\title{
Saint Domingue : Place d'Armes française dans les Antilles
}

\section{Nemours}

Volume 1, numéro 1, juin 1947

URI : https://id.erudit.org/iderudit/801348ar

DOI : https://doi.org/10.7202/801348ar

Aller au sommaire du numéro

\section{Éditeur(s)}

Institut d'histoire de l'Amérique française

\section{ISSN}

0035-2357 (imprimé)

1492-1383 (numérique)

Découvrir la revue

Citer cet article

Nemours (1947). Saint Domingue : Place d'Armes française dans les Antilles. Revue d'histoire de l'Amérique française, 1(1), 94-100.

https://doi.org/10.7202/801348ar d'utilisation que vous pouvez consulter en ligne.

https://apropos.erudit.org/fr/usagers/politique-dutilisation/ 


\section{SAINT DOMINGUE : PLACE D'ARMES FRANÇAISE DANS LES ANTILLES}

Le " Fait Français ) s'est manifesté de diverses façons dans les Antilles. L'une de ses plus magnifiques manifestations le fut dans le domaine des Armes. C'est elle que je voudrais rappeler. Elle est un éternel objet d'étude et d'admiration. Elle demeure un haut enseignement où, jusqu'aujourd'hui, nous pouvons tous puiser de salutaires et réconfortantes leçons.

Le 2 Décembre 1640, Monsieur de Poincy, Gouverneur de Saint Christophe, écrivait au Cardinal de Richelieu: "S'il plait à Votre Eminence me confier le gouvernement de cette Ile (La Tortue, qu'il appelle la Citadelle de Saint Domingue) je ferai mon possible de la conserver en attendant qu'elle trouve à propos de s'en servir pour la conquête de Saint Domingue ".

Le 13 Janvier 1669, M. Du Casse, Gouverneur de Saint-Domingue, mandait au Ministre de la Marine: " Je ne regarde pas cette Colonie de Saint Domingue pour la culture du sucre, indigo et tabac.s. Mais comme une place d'armes pour unir à la Monarchie française les importantes clés du Mexique, du Pérou et du Royaume de Santa Fé »).

L'on comprend que la Monarchie française ait accumulé dans ces îles de la Tortue et de Saint-Domingue de nombreuses fortifications et y ait organisé et entretenu de nombreuses troupes de toutes armes. Elle voulait faire de ces îles de puissantes et imprenables places d'armes. Elle y réussit malgré les nombreuses guerres qu'elle eut à soutenir dans ces parages.

1. Ces pages du Général Nemours, de Port-au-Prince, Haïti, sont extraites du 2e tome, encore inédit, de: Histoire militaire de Haiti, de 1640 à 1803 . Les documents qui suivent l'article sont des copies de pièces officielles: 1ère Garnison française à la Martinique en 1664; 1er Conseil de Guerre à la Guadeloupe en 1646; Commission datée de 1656 du ler Commandant de La Tortue; Ordre daté de 1666 pour l'embarquement des 1ères Troupes envoyées aux Isles; Brevet daté de 1683 du ler Major de la Tortue.

(N.D.R.) 
Les premières batailles qui furent livrées par des soldats français dans les îles des Antilles, le furent dans l'Ile de La Tortue. Le 31 Août 1643, le Capitaine Le Vasseur, à la tête de 49 hommes, quitta la Côte de Port Margot et débarqua à La Tortue. Après un sanglant combat, dans lequel mourut le fameux corsaire anglais Willis, Le Vasseur s'empara de La Tortue. Malgré des retours offensifs des adversaires, il se maintint dans l'île. La Tortue devint ainsi le premier établissement français à Saint Domingue, dont elle est une île adjacente. En 1653, le Capitaine Le Vasseur mourut assassiné par deux des membres du Gouvernement qu'il avait organisé. En 1656, le Chevalier de Fontenay fut le premier Gouverneur pour le Roi de La Tortue et Coste Saint Domingue.

Un des principaux Lieutenants de Le Vasseur, Jérémie Deschamps, Sieur de Moussac et de Rausset, fut commissionné par le Roi, Commandant de la Tortue, le 28 Novembre 1656. Sa Commission lui donnait: " la Garde de ladite Isle de La Tortue et des Forts qui y sont ou pourront être cy-après établis... avec pouvoir de commander, tant aux Habitants de ladite Isle qu'aux Gens de guerre qui y. sont ou seront ci-après établis en garnison... faire vivre lesdits habitans en union et concorde les uns avec les autres, contenir lesdits Gens de guerre en bon ordre et police suivant nos règlements... Ordonnons en outre, tant auxdits habitants qu'auxdits Gens de Guerre, de vous obéir et entendre ès choses touchant et concernant le présent pouvoir...) Une troupe française se trouvait donc à La Tortue avant 1656 où, bien avant cette date, il y avait eu de sanglantes batailles.

Les Français, après La Tortue, s'installèrent à la Guadeloupe et à la Martinique.

Le 1er Août 1646, un conseil de guerre fut installé à Basse Terre, localité de la Guadeloưpe. Il comprenait les principaux officiers de Milice. Et ce 1er Août, afin de faire reconnaître son autorité, le Sieur Patrocle de Thoisy, nommé Gouverneur Général, fit lire devant les Habitants et devant les Compagnies les Provisions Royales, le nommant Lieutenant Général.

D'après l'État de Dépense du 25 Octobre 1664, la garnison de la Martinique, comprenait: le Gouverneur et le Lieutenant de Roi, un chirurgien, un canonnier, un armurier, deux sergents, deux caporaux et 24 soldats. Ajoutons-y les valets du Gouverneur et du Lieutenant qui sont, ce que nous appelons, leurs Ordonnances. Les pre- 
miers contingents pris parmi les troupes réglées qui furent envoyés de France aux Isles de l'Amérique, et dont nous possédons un document officiel, le furent d'après un Ordre du Roi, daté du 24 Mars 1666. Ces contingents avaient été constitués par quatre compagnies du Régiment d'Infanterie de Poitou. Ils s'embarquèrent à La Rochelle. Ils débarquèrent à La Martinique en 1667.

Les premiers soldats Français envoyés de France pour tenir garnison à Saint Domingue, arrivèrent en 1688. Au nombre de 42, ils devaient former la Garde d'Honneur du Gouverneur, M. de Cussy. Ils furent installés à Port-de-Paix, siège du Gouvernement, et première Capitale de la Colonie de Saint-Domingue.

Les troupes débarquées de France provenaient généralement des compagnies du détachement de la Marine, servant à la garde des Ports Maritimes des Isles de l'Amérique. Une Ordonnance du Roi du 25 Août 1687, prescrivait aux Gouverneurs Particuliers de faire faire tous les mois des revues de ces compagnies. Ces revues devaient permettre de se rendre compte de leur effectif, de leur instruction, de leur santé.

En 1690, 82 soldats qui formaient la garnison de Saint-Christophe, furent débarqués à Port-de-Paix. Ils avaient été chassés de Saint-Christophe, dont les Anglais venaient de s'emparer.

Les pertes subies par les contingents stationnés ultérieurement à Saint-Domingue, décidèrent, le 23 Octobre 1694, le Roi Louis XIV à signer l'Ordonnance suivante: "Sa Majesté s'étant fait représenter les dernières Revues qui ont été envoyées des Compagnies qui sont en garnison danis les Forts des Isles Françaises, de l'Amérique, elle a trouvé que, quelque soin qu'on ait pris d'y envoyer de fréquentes recrues, pour les entretenir complètes, elles sont tellement diminuées qu'à peine la plus forte a-t-elle les deux-tiers des soldats qu'elle doit avoir et la plupart n'en ont que la moitié; ce qui ne peut être que l'effet du peu d'application que les Capitaines, qui ne sont pas chargés des Recrues ont à empêcher les soldats de déserter; à quoi, voulant pourvoir, S. M. a ordonné et ordonne, veut et entend qu'à l'avenir les Capitaines qui commandent les Compagnies qui servent dans les Isles de l'Amérique, seront chargés de remplacer ceux de leurs soldats qui déserteront ".

La désertion n'était pas la seule cause de la diminution des effectifs. La cause principale était la mortalité très élevée. Afin de la 
diminuer et de l'enrayer, il fallait acclimater les soldats nouvellement arrivés et, en cas de maladie, leur procurer des soins immédiats et effectifs. C'est ce que préconise la Lettre que le 25 Décembre 1697, le Ministre de la Marine et des Colonies, M. de Pontchartrain, adressa à M. Ducasse, Gouverneur de Saint-Domingue: "Le nombre de soldats nouveaux qui seront envoyés à Saint Domingue pour rendre les compagnies complètes, lui mandait-il,... m'oblige à vous faire part de la crainte que j'ai que ce secours ne soit pas aussi utile à la colonie qu'il est à désirer, par les maladies dans lesquelles tombent nécessairement ceux qui ne sont point accoutumés au climat de Saint-Domingue, qui en fera périr la meilleure partie, si on ne leur prépare des secours par lesquels ils puissent éviter les suites de ces premiers accidents. Pour y parvenir, il me parait que le soin le plus pressant est de penser à établir un hôpital au Cap et un autre à Léogane. " L'Hôpital du Cap confié aux Religieux de la Charité, venus de France, fut ouvert le 1er Août 1698.

L'Hôpital de Léogane, confié à deux Religieux de la Charité, envoyés de la Guadeloupe, fut installé en 1700.

En 1703, l'Intendant, M. Deslandes augmenta le nombre des Compagnies Militaires, stationnées à Saint-Domingue et le porta à dix.

Le 20 Avril 1713, le Règlement du Roi organisa la Première Compagnie d'Artillerie qui fut destinée à l'Ile de La Tortue. Elle comprenait 60 hommes et 2 officiers. Elle était commandée par M. de la Grange, enseigne d'infanterie en garnison à La Tortue, qui fut nommé eapitaine.

Pendant de très nombreuses années, Saint Domingue fut associé aux gloires militaires de la France. Depuis 1697 puisque les troupes françaises qui débarquèrent devant Carthagène, comprenaient un important contingent levé à Saint-Domingue: troupes réglées, Habitants, Volontaires de toutes couleurs. C'est la Compagnie de Volontaires Noirs de Saint-Domingue qui s'empara du Fort de la Bocachica, clef de la défense. Le contingent de Saint-Domingue s'illustra sur la Terre Ferme pendant la Guerre de l'Indépendance des États-Unis.

Le nom de Saint-Domingue demeure toujours associé aux fastes militaires de la France. Le 106ème Régiment d'Infanterie de l'Armée Française était le Régiment du Cap-Français, formé en 1773. Le Régiment de Saint Pouange Cavalerie s'appela en 1728 le Régiment de Bongars-Cavalerie, lorsqu'en 1721 Pierre de Bongars, neveu du 
Gouverneur de Saint Domingue, en fut le Colonel. Bongars-Cavalerie devint le 21ème Régiment de Chassours à Cheval de l'Armée Fran.çaise.

En boutant hors de chez elle les envahisseurs, en aidant à triom-pher, les troupes françaises envoyées au secours des Insurgents Américains, Saint-Domingue a vaillamment rempli son rôle de "Place d'Armes ". A cette gloire militaire, Haïti ajoute une gloire intellectuelle. Elle conserve précieusement le dépôt de la culture française, puisqu'elle est la seule nation indépendante des Amériques dont la langue officielle est le Français.

\section{Général Nemors Ris.}

Documents annex es as pricédent arible.

GARNISON FRANÇAISE A LA MARTINIQUE, d'après l'Hxtrait de l'Eitát de dépense du 25 octobre 1664 .

Gouverneur. Lieutenant de Roi, 1 Chirurgien, 1 Canonnier, 1 Armurier, 24 Soldats, 2 Caporaux, 2 Sergents; valets du Grouverneur, valets du Lieutenant.

ORDONNANCE DE M. PATROCLES'DE THOLSY, Gouverneur Général des Isles portant Etablissement d'un Conseil de Guerre au Quartier de la Bassaterre, Guadeloupe. Du ler anout 164t).

Le Sieur de Thoisy, Chevalier... nous avons fait faire lecture Dimanche 21 Juillet dernier, en ce quartier, de la Basseterre, à la tête des Compagnies, de nos provisions de la Charge de Lieutenant-Général, et ensuite de ce, jugé à propos de faire assembler les Compagnies de la Capesterre, et faire aussi lecture sur l'original de nos dites provi ions... nous avons estimé nécessaire pour le service du Roi ế l'utilité publique, d'établir un Conseil de guerre, qui sera composé des principaux: Officiers de Milice, avec tels autres que nous jugerons à propos, lequel Conseil se, tiendra tous les premiers Dimanches des mois, après le Service Divin, au Fort de l Basseterre de cette Isle, lieu de notre résidence, à commencer le Dimanche, cin. quième Août... Nous avons nommé et commis le Sieur de Boisfaye, notre Capitaine des Gardes, pour icelle faire lire et publier à la tête des Compagnies, le Dimanche cinquième Août, afin que personne n'en prétende cause d'ignorance.

Fait au fort de la Basseterre de la Guadeloupe, le ler Aont 164t).

Signé De Thoisy et contresigné pa. Loyer, Secrétaire. 
COMMISSION DU PREMIER COMMANDANT POUR LE ROL DF, L'ISLE DE LA TORTUE, M. JËREMIE DESCHAMPS, SIEUR DE MOUGSAC et DE RAUSSET. 26 Novembre 1656.

Louis, etc. A notre cher et bien aimé Jérémie Deschamps, Sieur de Maussac et de Rousset, Salut. Ayant jugé nécessaire de pourvoir à la garde et conservation. de l'Isle de la Tortue en l'Amérique nouvellement remise sous notre obéissance, et d'y établir quelque personnage sur la suffisance et fidélité duquel nous puissionis nous en reposer, et qui prit un soin particulier du rétablissement des Forts qui $y$ étaient ci-devant et qui ont été ruinés par les Ennemis de cet État, en sorte qu'ils fussent suffisans, tant pour la sûreté des Habitans de l'Isle que de ceux de nos Sujets qui pourraient y aller faire de nouvelles habitations, ainsi qu'ils ont fait par le passé; nous avons à cette effet jeté les yeux sur vous dans la créance que nous avons eue que nous ne pouvions faire choix de personne qui pât mieux que vous s'acquitter de cet emploi vu votre expérience et votre valeur dont vous avez donné de si notables preuves en la reprise de la même Isle; et ayant d'ailleurs une entière confiance en la fidélité et en l'affection que vous avez fait paraître en toute ren.contre à netre service.

A ces causes, et autres à ce nous montrant, nous vous avons commis et ordonné, commettons et ordonnons par ces présentes signées de notre main pour, sous notre autorité et celle des Gouverneurs et nos Lieutenants-Généraux ès Isles de l'Amé. rique, avoir la garde de ladite Isle de la Tortue et des Forts qui y sont ou pourront être ci-après établis, et de ladite charge jouir aux honneurs, autorités, prérogatives, prééminences, droits, appointements, profits et émolumens, tels et semblables qu'en ont joui ou dâ jouir ceux qui vous ont précédé en la même charge, avec pouvoirs de commander, tant aux Habitans de ladite Isle qu'aux Gens de guerre qui y sont ou seront ci-après établis en garnison, ce qui sera du bien de notre Service, faire vivre. lesdits Habitans en Union et Concorde les uns avec les autres, contenir lesdits gens de guerre en bon ordre et police suivant nos Règlemens, maintenir le commerce ec trafic de ladite Isle, et généralement faire, tout ce que vous jugerez à propos pour la sûreté et la conservation d'icelle, tant qu'il nous plaira: de ce faire vous avons donné et donnons pouvoir, commission et mandement spécial par cesdites présentes par lesquelles mandons à tous Gouverneurs et nos Lieutenans-Généraux ès Isles de l'Amérique de vous faire reconnaître en ladite qualité par tous ceux qu'il appar.tiendra; ordonnons en outre, tant auxdits Habitans qu'aux dits Gens de Guerre, de Vous obéir et entendre ès choses touchant et concernant le présent pouvoir: (3, tel est notre plaisir, etc.

Donné à Paris, le vingt sixième jour de Novembre, l'an de grâce mil six centí cinquante six, et de notre règne le dix huitième.

Ordre du Roi du 24. Mars 1666, pour l'Embarquement des Premières 'Truupes: réglées par Lui envoyées aux Isles. 
- S. M. ordonne aux quatre Compagnies du Régiment d'Infanterie de Poitou qui se doivent rendre du Côté de la Rochelle, de s'embarquer sur les vaisseaux de S. M. dans le temps et ainsi qu'elles en seront requises par le Sieur Colbert de Tenon, Intendant au Pays d'Aunis, et Isles adjacentes, pour passer aux Isles Occidentales, et y étant, faire tout ce qui leur sera ordonné par le Sieur de la Barre, Commandant sur les Vaisseaux de S. M. du côté desdites Isles, avec assurance que les services qu'elles y rendront lui seront très agréables.

Fait à St-Germain-en-Laye, le 24 Mars 1666.

Signé Louis, et plus bas Le Tellier.

Brevet du premier Major pour le Roi de l'Isle de la Tortue et Côte de Saint Domingue. Du 5 Novembre 1683, " pour le Sieur le Clerc de la Boulays...

sous l'autorité du Gouverneur Particulier de ladite Isle de la Tortue et Côte Saint Domingue. »

Le Sieur le Clerc de la Boulays fut le premier major pour le Roi, nommé et commissionné. M. Renon depuis 1671, n'était que Major des Milices sous Commission.

Provisions de Lieutenant de Roi de la Côte Saint-Domingue pour M. Dumas. 6 Aout 1685.

Ce fut la seconde Lieutenance de Roi établie à Saint-Domingue après la mort de M. de Cussy survenue à Limonade en 1691, dans un combat contre les Espagnols. M. Dumas qui était Lieutenant de Roi au Gouvernement de l'Isle de la Tortue et Côte Saint-Domingue à la résidence du Quartier du Cul-de-Sac, devint Commandant pour toute la Partie Française. 